\title{
Genetic interactions underlying flower color patterns in Antirrhinum majus
}

Jorge Almeida, Rosemary Carpenter, Timothy P. Robbins, ${ }^{1}$ Cathie Martin, and Enrico S. Coen ${ }^{2}$

John Innes Institute and AFRC Institute of Plant Science Research, Colney Lane, Norwich NR4 7UH, UK

Diverse spatial patterns of flower color in Antirrhinum can be produced by a series of alleles of pallida, a gene encoding an enzyme required for pigment biosynthesis. The alleles arose by imprecise excision of a transposable element, Tam3, and we show that they carry a series of deletions involving progressive removal of sequences adjacent to the excision site. This has enabled us to define three cis-acting upstream regions, $A, B$, and $C$, which differentially affect the level of pallida expression in distinct areas of the flower. We show further that an unlinked locus, delila, regulates the spatial distribution of pallida transcript. Deletion of regions $\mathbf{A B C}$ at the pallida locus uncouples pallida from regulation by delila, whereas deletion of $\mathrm{A}$ or $\mathrm{AB}$ brings pallida under regulation by delila in a new area of the flower. These results suggest that diverse patterns of pallida expression reflect the different ways in which alleles interact with a prepattern of both common and spatially specific genetic signals in the flower.

[Key Words: Allelic series; gene regulation; transposons; plants]

Received June 16, 1989; revised version accepted August 22, 1989.

The molecular mechanisms by which multiple alleles at a single locus confer diverse phenotypic patterns are poorly understood. In most cases analyzed, the alleles are of genes encoding products that do not readily diffuse between cells. Diverse patterns are thought to be the result of differences in the way alleles interact with or interpret pre-existing patterns, termed prepatterns (Stern 1954,1968 ). Molecular analysis of this interaction has been difficult because the genetic determinants of the prepattern are unknown in many of the cases, such as yellow or scute in Drosophila, where different alleles have been isolated (Campuzano et al. 1985; Chia et al. 1986). Furthermore, many of the alleles carry transposon insertions, confounding the regulation of the host gene with that of the transposon (Geyer et al. 1988). Perhaps the best study to date has been on the white ${ }^{\text {spotted }}$ alleles in Drosophila, which change the distribution of pigment between testis and eye and uncouple white from inhibition by the zeste $e^{1}$ mutant allele (Davison et al. 1985; Pirrotta et al. 1985). Here, we investigate the structure and expression of a series of alleles of the pallida (pal) locus conferring diverse patterns of flower color in $A n$ tirrhinum majus and how they interact with a regulatory gene delila (del). A particular advantage of studying the pal alleles is that they arose by imprecise excision of

1Present address: Department of Biology, Yale University, New Haven, Connecticut 06511.

${ }^{2}$ Corresponding author. a transposon, permitting a systematic study of a regulatory region of the pal gene, independently of the transposon (Coen et al. 1986).

The pal gene encodes a product required for the synthesis of red anthocyanin pigment in flowers (Stickland and Harrison 1974). Plants in which the expression of pal is completely blocked have ivory, rather than full red, flowers. An unstable recessive allele, pal-2 (pallidarecurens), carries the transposable element Tam3 inserted near the pal promoter, preventing pal transcription (Martin et al. 1985; Coen et al. 1986). Somatic excision of Tam3 during flower development gives rise to clones of pigmented cells on an ivory background, indicating that pal acts in a cell-autonomous manner. Excision of Tam3 in the germ line produces stable alleles, which give either the wild-type phenotype (full red) or altered intensities and patterns of pigmentation (Baur 1924; Fincham and Harrison 1967). Analysis of some of these pal alleles has revealed that they have nucleotide sequence alterations at the site of Tam 3 excision (Coen et al. 1986).

Here, we describe a set of $p a l$ alleles with distinct patterns of expression that result from a series of deletions involving progressive removal of sequences adjacent to the Tam 3 excision site. We show that an unlinked locus, del, regulates the spatial distribution of pal transcript. The pal deletions either uncouple pal from regulation by del or alter the spatial specificity of the del effect. The resulting patterns of pal expression can be explained by 
the different ways in which the alleles interact with a prepattern of both common and spatially specific genetic signals in the flower.

\section{Results}

\section{Patterns of pal expression}

The pal gene encodes dihydroflavonol-4-reductase, an enzyme that catalyzes the reduction of dihydroquercetin to leucocyanidin, a late step in the anthocyanin biosynthetic pathway (Kristiansen 1984; Heller et al. 1985). This was concluded from precursor feeding experiments in which pigment synthesis was observed when flowers of pal mutants were imbibed with leucocyanidin but not when they were supplied with dihydroquercetin. As a control, flowers of a mutant at incolorata (inc), a gene that acts at an earlier step than pal in the anthocyanin pathway (Stickland and Harrison 1974; Forkmann and Stotz 1981), were imbibed with either leucocyanidin or dihydroquercetin; in each case, pigment synthesis resulted. Further evidence for the biosynthetic role of the pal product comes from the homology between the sequence of amino acids encoded by the first exon of pal and that of the $A 1$ gene of maize which also encodes dihydroflavonol-4-reductase (Coen et al. 1986; SchwarzSommer et al. 1987).

Most of the stable alleles derived from the unstable allele pal-2 confer the wild-type phenotype (Fig. 1; pal-501), in which, during flower development, anthocyanin synthesis occurs first in a ring at the base of the

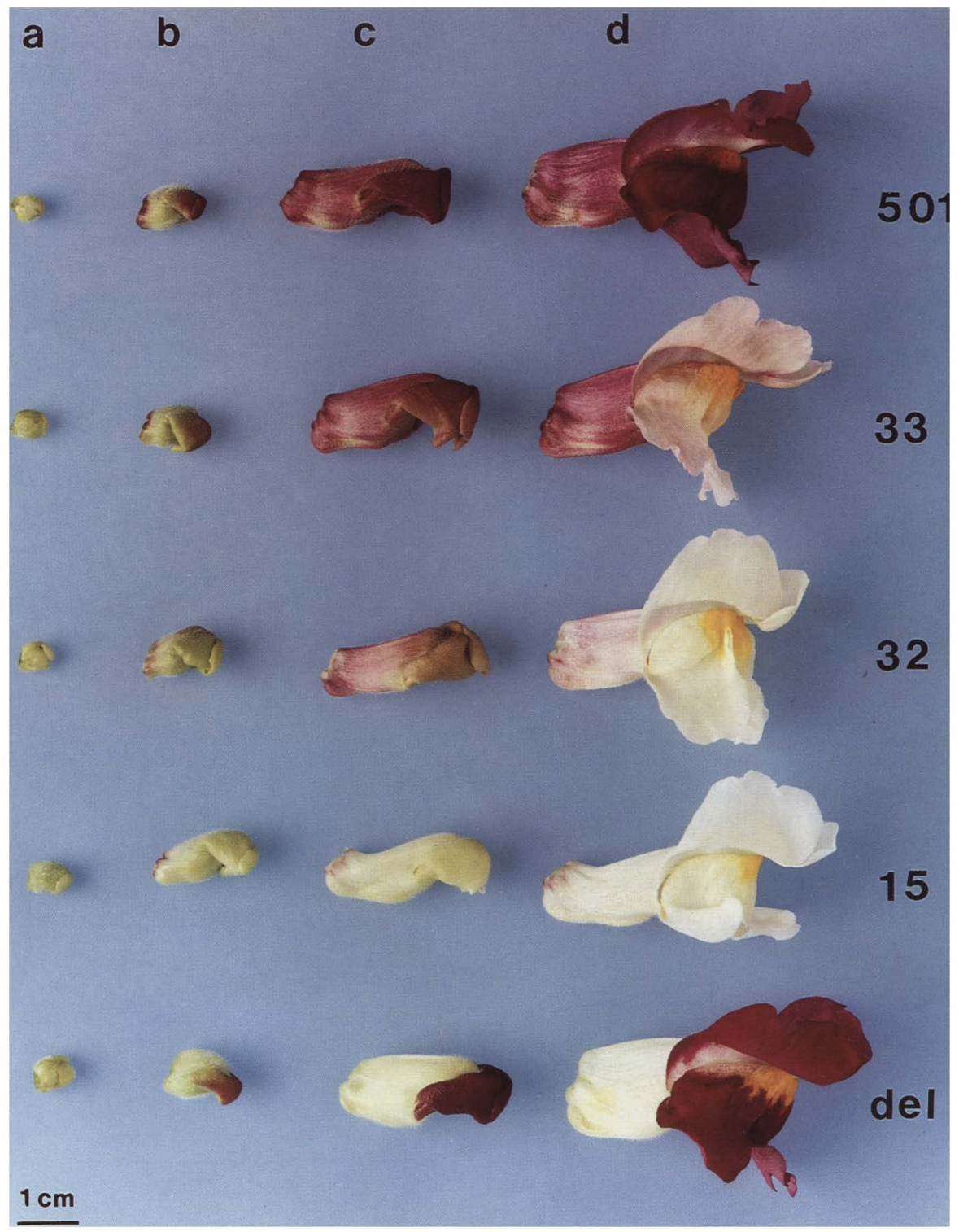

Figure 1. Phenotypes conferred by various pal alleles $\{501,33,32$, and 15$\}$ and a mutation at the del locus, at several stages of flower development. Alleles conferring similar phenotype to pal-33 include pal-518 and pal-35G. The pal-15 allele confers s similar phenotype to pal-569. 
tube and in the lobes (Fig. 1; pal-501, stage b). In the next stage (c), the tube becomes fully pigmented, giving a pattern that is maintained through to the final stage of development (d). Thus, anthocyanin synthesis appears to follow a temporal and spatial pattern that defines three areas in the flower corolla: the base of the tube, the area of the tube in which pigment synthesis occurs at a late stage, and the lobes. The pal alleles that confer different relative intensities of pigmentation in these three areas can be divided into three classes. The first class includes pal-15 and pal-569, which give color confined to the ring at the base of the tube that appears at stage $b$, the rest of the corolla remaining unpigmented (Fig. 1). The second class contains pal-32, which gives palely colored tubes and almost unpigmented lobes. This type of pattern is of particular interest because it is the inverse of that produced by mutations at the del locus, which is unlinked to $\mathrm{pal}$ and gives flowers with red lobes and unpigmented tubes (Wheldale 1907; Fig. 1). Alleles of the third class (pal-33, pal-35G, and pal-518) give pale color to the lobes and almost wild-type pigmentation in the tubes (Fig. 1; Table 1).

It was shown previously that alleles of all three classes (pal-15, pal-32, pal-33, and pal-518) produce reduced overall levels of pal transcript relative to wild type (Coen et al. 1986). Two explanations may be proposed for how changes in pal transcription can result in altered patterns of color. One is that diverse pigmentation patterns reflect changes in the spatial distribution of pal transcript. The other is that different threshold levels must be reached in distinct areas of the flower before a change in transcription results in altered pigment production. In this case, overall reductions in pal transcription without change in its spatial distribution could result in different patterns of color.

To determine the spatial distribution of transcript conferred by diverse pal alleles, Northern and slot-blots of RNA extracted from different areas of flower buds were probed with a pal clone. More pal transcript was detected in the lobes than in the tubes in pal-501 and pal-520, whereas less transcript was produced in the lobes than in the tubes in pal-518, pal-33, and pal-32
(Table 1; Fig. 2a,b). The quantity of pal mRNA at the base of the tube of pal-15 was reduced to $\sim 1 \%$ of that in the pal-520 control in the same area (Fig. 2c). No transcript was detected in the remainder of the corolla for pal-15, whereas higher levels of pal mRNA were found in the rest of the corolla than at the base of the tube in pal-520 (Fig. 2c). These results show that distinct patterns of color conferred by the pal alleles reflect different spatial distributions of pal mRNA. As a control, the same blots were probed with a clone of the nivea (niv) gene that encodes chalcone synthase, an enzyme also required for anthocyanin synthesis (Wienand et al. 1982). The levels and spatial distributions of niv transcript were similar, irrespective of pal genotype (Table 1; Fig. 2). In another control, the relative levels of pal and niv transcripts produced in the upper and lower halves of the corolla in flowers from the lines containing pal-501 or pal-518 were determined. Transcripts of pal and niv were found to be distributed evenly between the two areas in both lines (Fig. 2d). Transcription initiation was also investigated to determine whether pal mRNA detected in blots was accurately initiated. S1 mapping of RNA produced by pal-501, pal-518, pal-33, pal-32, and pal-15 revealed that the sites of transcription initiation in all of these alleles were identical to those found previously in $\mathrm{Pal}^{+}$and other mutant alleles (Coen et al. 1986; Robbins et al. 1989; data not shown).

\section{Structures of pal alleles}

It has been shown previously by Southern blots that many of the pal alleles arose by imprecise excision of Tam3 from the pal-2 allele (Coen et al. 1986). The regions around the excision site were sequenced for each allele (Fig. 3). In pal-501 and pal-520, both of which confer wild-type phenotype, sequence changes were confined to nucleotides of the 5-bp target duplication of pal sequence that flanked Tam 3 in the pal-2 progenitor. In pal-520, 4 bp of each of the 5-bp target copies was retained and flanked a 4-bp inverted duplication of target sequence. The net results of these changes were insertions of $1 \mathrm{bp}$ in pal-501 and $7 \mathrm{bp}$ in pal-520, relative to

Table 1. Levels of pal and niv mRNAs in tubes and lobes of flowers carrying the pal alleles indicated

\begin{tabular}{|c|c|c|c|c|c|c|c|c|c|}
\hline \multirow[b]{3}{*}{ Allele } & \multirow{2}{*}{\multicolumn{3}{|c|}{ Anthocyanin }} & \multicolumn{6}{|c|}{ mRNA } \\
\hline & & & & \multicolumn{3}{|c|}{ pal } & \multicolumn{3}{|c|}{ niv } \\
\hline & $\overline{\mathrm{T}}$ & $\mathrm{L}$ & $\overline{T / L}$ & $\overline{\mathrm{T}}$ & $\mathrm{L}$ & $\mathrm{T} / \mathrm{L}$ & $\overline{\mathrm{T}}$ & $\mathrm{L}$ & $\mathrm{T} / \mathrm{L}$ \\
\hline 501 & 100 & 440 & 0.25 & 100 & 200 & 0.5 & 100 & 420 & 0.24 \\
\hline 520 & 110 & 470 & 0.25 & 130 & 190 & 0.7 & 130 & 400 & 0.32 \\
\hline 518 & 100 & 130 & 0.80 & 25 & 20 & 1.2 & 120 & 430 & 0.28 \\
\hline 33 & 60 & 50 & 1.20 & 25 & 10 & 2.5 & 160 & 420 & 0.37 \\
\hline 32 & 10 & 5 & 2.00 & 4 & 1 & 4.0 & 160 & 420 & 0.37 \\
\hline
\end{tabular}

All measurements are relative to the levels detected in the tubes of pal-501, which confers wild-type phenotype. T/L is the ratio of the levels in tubes and lobes. Alleles pal-501, pal-520, and pal-518 are maintained in isogenic backgrounds because they were derived by selfing a common progenitor containing pal-2, which has been inbred for many generations. Allele pal-518 had previously been considered to have a uniform reduction in pigmentation relative to wild type. However, as shown here and in Fig. 3 , the level of pigmentation in the lobes is much more reduced than in the tubes. Alleles pal-33 and pal-32 were selected in progenies of crosses between lines containing pal-2 and other lines carrying mutant pal alleles. The S.E.M., based on five independent measurements, is $10-20 \%$ for the anthocyanin levels shown. The levels of pal and niv mRNAs were determined by slot-blot analysis. 
a

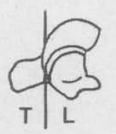

b

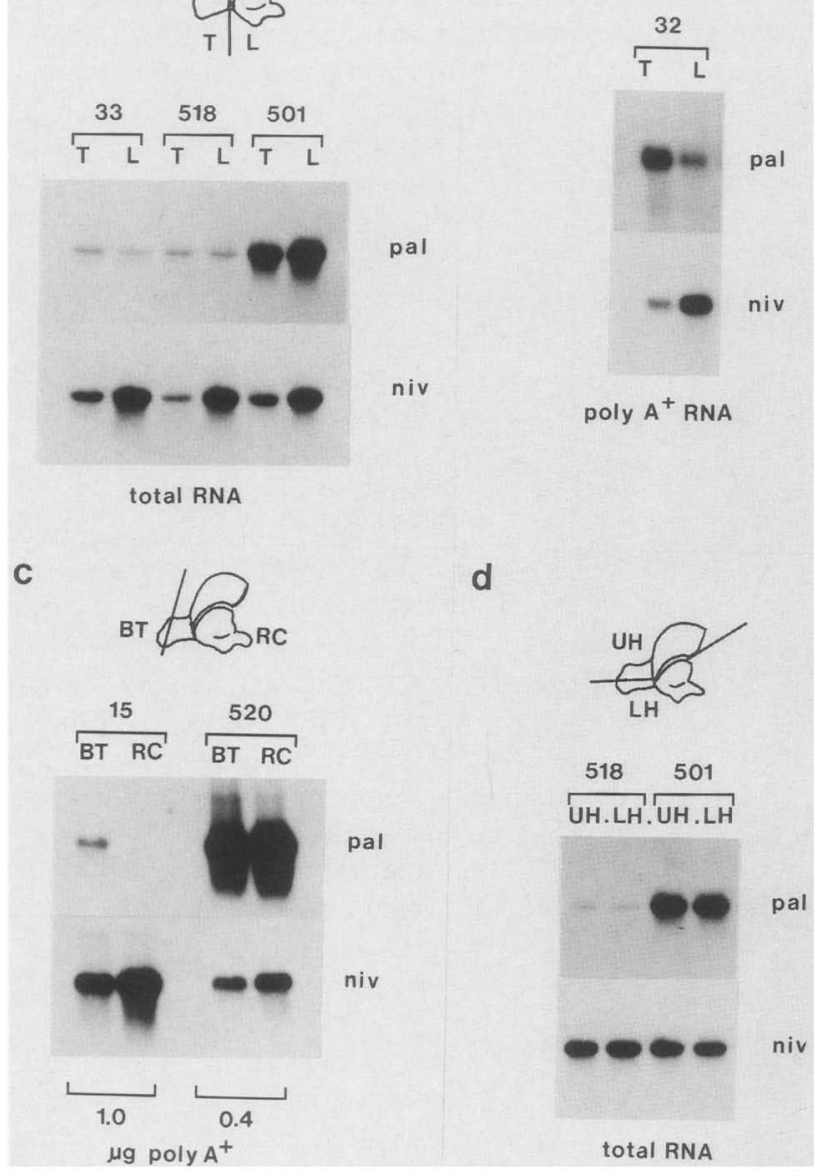

Figure 2. Spatial distribution of pal and niv transcripts in flowers containing the pal alleles. Numbers above the blots indicate the pal alleles analyzed. Northern blots were first probed with a clone of the pal gene and then with a clone of niv. RNA was from flowers dissected as represented in the diagrams shown above the blots. The areas from which RNA was extracted are indicated above each lane: (T) Tubes; (L) lobes; (UH) upper half of the corolla; (LH) lower half of the corolla; (BT) base of the tube; $(\mathrm{RC})$ rest of the corolla. The high intensity of the signal produced by pal-520 (in c) does not allow the differences in the levels of pal mRNA in BT and RC to be seen. A shorter exposure and slot-blot analysis revealed that in this case, the intensity of the signal obtained in RC was twofold higher than that in BT.

wild-type (pal-18). This suggests that normal levels of pal expression are compatible with the disruption of the Tam 3 target sequence and small increases in the spacing between the regions to its left and right.

The three types of phenotypes conferred by mutant alleles correlate with progressive deletion of three regions to the left of the Tam3 excision site. Compared with pal-501, alleles pal-33, pal-35G, and pal-518 carry deletions of 7,13 , and $14 \mathrm{bp}$. The similar phenotype conferred by these alleles suggests that it results from deletion of a common 7-bp sequence, region A (Fig. 3). Dele- tion of region $\mathrm{A}$ and a further 9-10 bp to its left (region B), results in reduced expression of pal-32. Deletion of regions $\mathrm{AB}$, together with an additional $79 \mathrm{bp}$ to the left (region $\mathrm{Cl}$, results in expression confined to the base of the tube (pal-15). Removal of 21 bp to the left of region C (in pal-569) has no further effect on the pattern of pal expression.

Although the mutant alleles described fall into three classes, there are minor differences in phenotype within a class (e.g., pal-33, pal-35G, and pal-518; Fig. 3). This raises the question of whether some of this variation is the result of the small differences in sequence between these alleles or to the genetic backgrounds of the lines in which they are maintained. Analysis of an $F_{2}$ population from a cross between the lines containing pal-33 and pal-518 showed that flower color varied in the range defined by the parental lines. Because pal-33 and pal-518 can be distinguished on Southern blots (see legend to Fig. 3), the genotypes of several plants from this $F_{2}$, showing similar phenotypes, were determined. This revealed that flower color did not correlate with the combinations of the two alleles, suggesting that the differences in phenotype between the parental lines were caused by differences in genetic background. This was further confirmed by $\mathrm{F}_{2}$ populations from intercrosses between lines carrying pal-501, pal-518, pal-33, pal-32, and pal-15. Phenotypes typical of the pal-518 parental line were often observed in crosses involving pal-33 and vice versa. Phenotypes typical of the pal-501, pal-32, and pal-15 parental lines were only observed when the corresponding alleles were used in the crosses, showing that genetic background was not responsible for the major phenotypic differences between the three classes of alleles.

The deletions and the inverted duplications in the various alleles can be readily explained by a hairpin model for the excision of Tam3 detailed elsewhere (Coen and Carpenter 1988; Coen et al. 1986, 1989). However, in pal-15, the mechanism by which a sequence normally located $125 \mathrm{bp}$ upstream of the Tam 3 target was directly duplicated at the junction of a 98-bp deletion and an inverted duplication remains unclear.

\section{del regulates pal expression}

Mutations at del give flowers with fully pigmented lobes and unpigmented tubes (Fig. 1). This is most probably the null phenotype because several independent del mutants, some of which are the result of transposon insertion, confer the same phenotype (R. Carpenter and E.S. Coen, unpubl.). To determine whether genes encoding enzymes of the pigment pathway were regulated by del, Northern and slot-blots of RNA from tubes or lobe of $\mathrm{Del}^{+}$or del flowers were probed with clones of either the pal or niv genes. The levels of mRNA from both genes in the lobes were unaffected by del (Fig. 4). However, the quantity of pal transcript was reduced $\sim 100$ fold and that of niv $\sim 5$-fold in the tubes of del relative to $\mathrm{Del}^{+}$flowers. These results show that the $\mathrm{Del}^{+}$gene product is specifically required in the tubes but not in 


\section{Almeida et al.}

the lobes for wild-type levels of niv and pal expression. Mutations at del also reduce the levels of inc transcript in flower tubes but have no effect in the lobes /C. Martin, unpubl.). In addition, the del gene regulates genes acting at other steps in the anthocyanin pathway because imbibition of flowers mutant for both pal and del with leucocyanidin resulted in additional pigment synthesis in the lobes but not in the tubes. This is con-

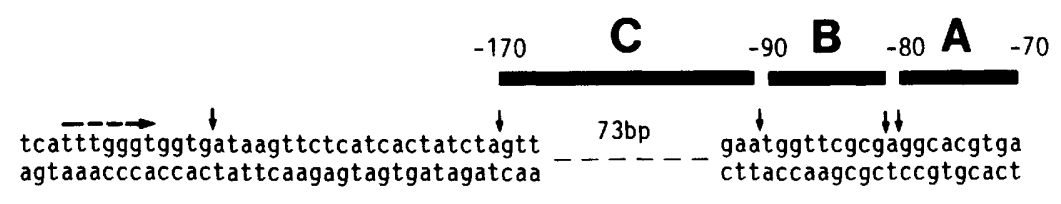

$\longrightarrow-----$
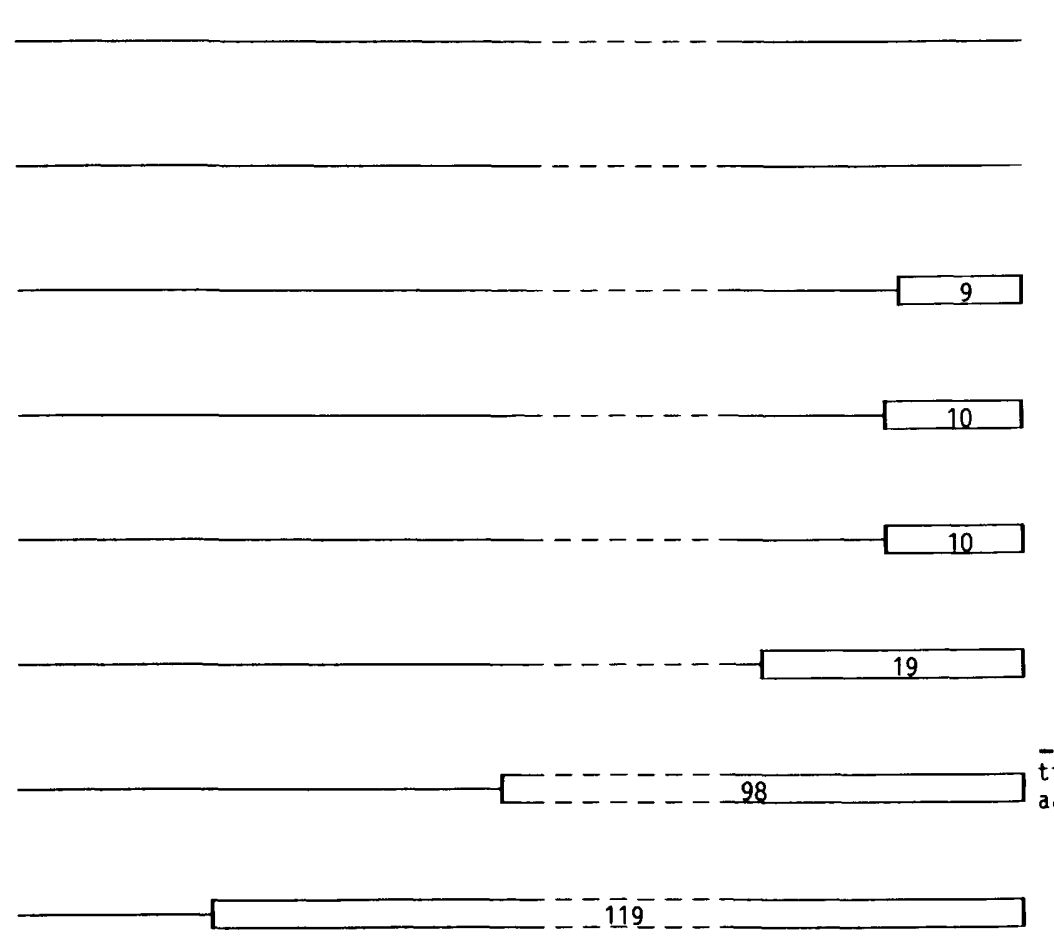

$\overrightarrow{t t t g g g t}$ GGTA $\overrightarrow{A C C C}$ aacCCa CCATETGGG

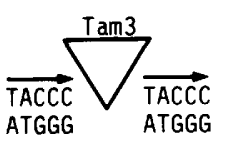

ATGGG
$\overrightarrow{C C}$

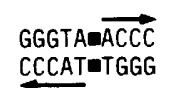

tacca atggt

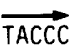
ATGGG

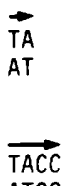

ATGG

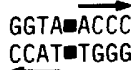
CCATUTGGG

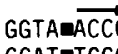
CCATETGGG

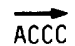

TGGG

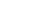

\section{(n)}

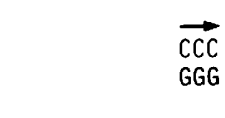

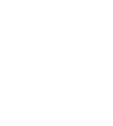
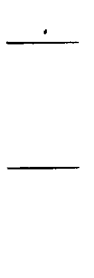

18

501

520

33

35G

518

\section{2}

15

569

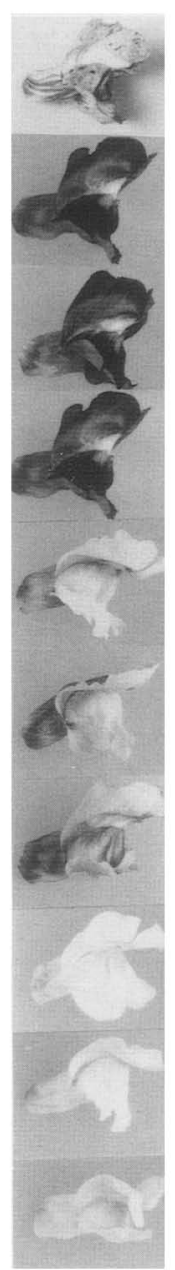

Figure 3. Structure of pal alleles. (Top) The pal-2 allele, which carries the transposable element Tam3. The wild-type allele, pal-18, comes from a commercial fully red line (see Coen et al. 1986). All other alleles shown have resulted from Tam3 excision. Nucleotides of the target duplication generated following integration of Tam 3 in pal-2 are represented by uppercase letters with horizontal arrows above. The sequences flanking these nucleotides are indicated by lowercase letters. Horizontal lines represent sequences that are identical in all alleles. For ease of illustration, 73 bp of sequence (dashed line) has not been shown in full. Open boxes represent deletions. Numbers inside the boxes indicate the sizes of the deletions (in bp) extending from the left end of the Tam 3 target. The left end points of these deletions are indicated by vertical arrows on the sequence of $\mathrm{pal}-2$. Solid squares in inverted duplications represent a nucleotide missing at the center of symmetry of what would otherwise be a perfect palindrome. In pal-33, the dinucleotide GG, indicated as part of an inverted duplication, might have been represented as part of the normal pal sequence, 8 bp to the left of the target. In this case, the deletion end point would have been $2 \mathrm{bp}$ to the right of the site indicated. Horizontal dashed arrows indicate a sequence duplicated in direct orientation in pal-15. (Top) Solid bars represent regions A, B, and C (see text for details). Numbers above these boxes indicate their positions relative to the most upstream pal transcription initiation site. The sequences shown were consistent with a number of differences between the alleles revealed by Southern analysis of genomic DNA. Alleles pal-520, pal-33, pal-32, and pal-15 carry the BstEII site (GGTNACC) generated by an inverted duplication of target nucleotides. The CCGG site, also generated at the Tam3 excision site in pal-520, was cleaved by HpaII. A NruI site, 10 bp to the left of the Tam3 target (TCGCGA), was present in pal-33 and pal-520 but not in pal-35G, pal-32, pal-15, or pal-569. In pal-15 and pal-569, the HindIII fragments containing the Tam 3 excision site were $\sim 100$ bp smaller than those from other alleles. The sequences of pal-2, pal-18, pal-501, and pal-518 were reported previously (Coen et al. 1986). 


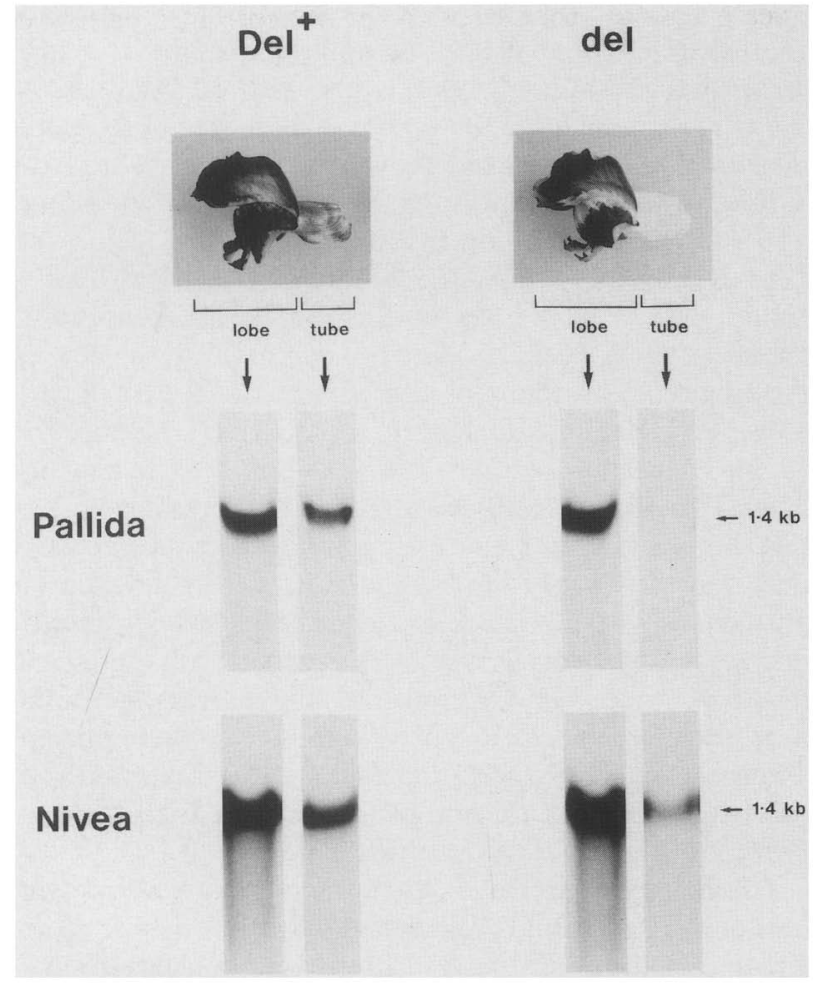

Figure 4. Effect of del on pal and niv expression. A Northern blot of total RNA extracted from the tubes or lobes of $\mathrm{DeI}^{+}$or del flowers was probed with pal and niv, as described in Fig. 2. $\mathrm{Del}^{+}$flowers were from a standard wild-type line (JI7). The del allele is from line JI8, which has an otherwise wild-type genotype.

sistent with the observation that the activity of UDPglucose : flavonoid-3-O-glucosyltransferase, an enzyme acting at a later step than dihydroflavonol-4-reductase, can be detected in the lobes but not in the tubes of del flowers (Martin et al. 1986).

\section{Interaction between pal and del}

The observed patterns of expression in different pal alleles may reflect altered interactions between the alleles and regulatory genes that affect pal expression in a spatially specific manner (Coen and Carpenter 1986). To investigate whether the pal alleles showed an altered interaction with the regulatory gene $d e 1$, their expression was analyzed in a $\mathrm{Del}^{+}$or del background. Because del regulates several steps in the pigment pathway, pal expression in tubes of del mutants will not result in pigment production. This allowed del mutants to be recognized easily by the lack of tube pigmentation, irrespective of the pal genotype (Fig. 5) but precluded the use of pigmentation as an assay for pal expression in the tube.

To determine the effect of the pal deletions on the interaction between pal and del, the levels of pal transcript were measured in tubes and lobes of $d e l$ and $\mathrm{Del}^{+}$ genotypes. Transcript levels were reduced in the tubes of pal-32; del relative to pal-32; $\mathrm{Del}^{+}$flowers, indicating that pal-32, which lacks regions $A B$, was still regulated by del (Fig. 6a). Interestingly, pal-32 expression in lobes was also clearly reduced in del mutants, resulting in complete abolition of pigmentation in lobes (Figs. 5 and 6a). This is in contrast to $\mathrm{Pal}^{+}, \mathrm{NiV}^{+}$, and $\mathrm{Inc}^{+}$alleles, the expression of which is not affected by del in the lobes (Figs. 4 and 6). The effect of del on the lobes was also observed in flowers containing pal-33 or pal-35G in which only region $\mathrm{A}$ is deleted. In these cases, del caused a threefold reduction in pigmentation levels in the lobes (Fig. 5). This indicates that in the absence of regions A or $\mathrm{AB}$, the spatial specificity of the effect of del on pal expression is altered.

In pal-15, which lacks regions $A B C$, the levels and spatial distributions of transcript were found to be similar, irrespective of the del genotypes (Fig. $6 \mathrm{~b}$; see also Fig. 2c). As a control, we showed that inc was regulated by del in pal-15 genotypes. This indicates that region $\mathrm{C}$ is required for the interaction between pal and del.

A previously described allele conferring a complex pattern of pigmentation, pal-41, also lacks regions $A B C$, because the entire region upstream of -70 has been re-

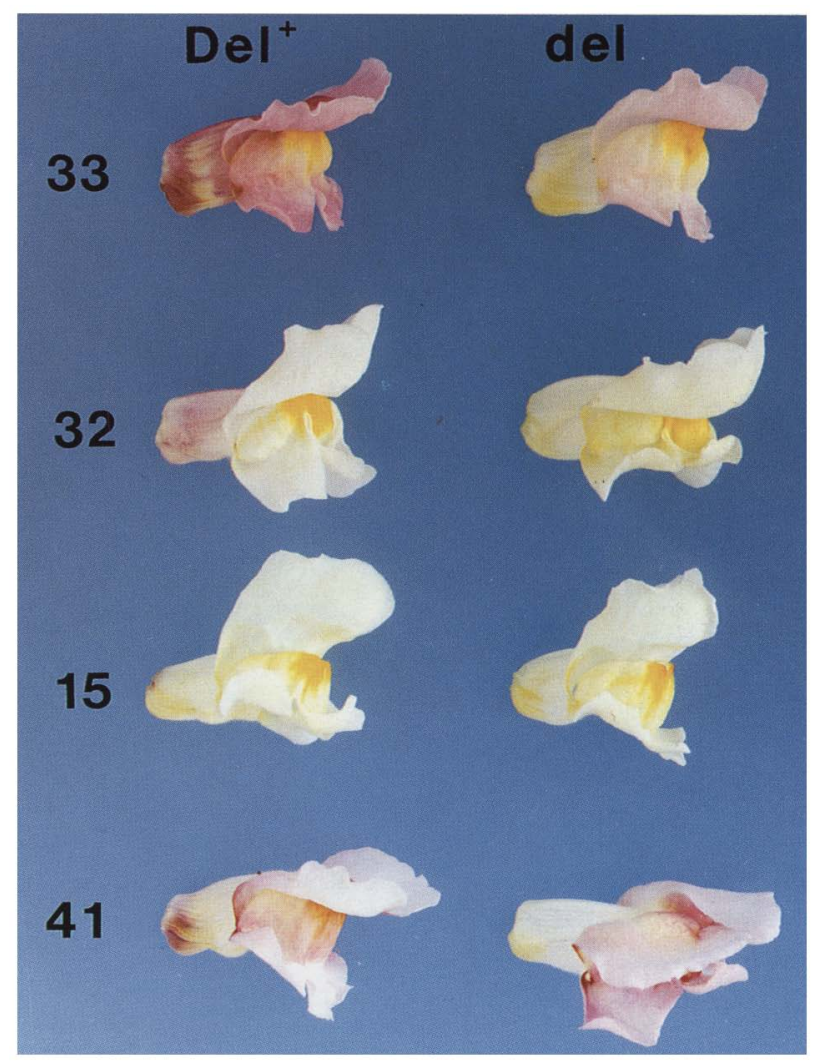

Figure 5. Phenotype conferred by pal alleles in a $\mathrm{Del}^{+}$or $\mathrm{del}$ background. Note that the lack of anthocyanin in the tubes of the del mutants does not reflect the level of pal expression (see text and Fig. 6). The difference in lobe pigmentation between pal-32; $\mathrm{Del}^{+}$and pal-32; del can be observed more easily at an earlier stage of flower development (stage c, Fig. 1). 
a

b

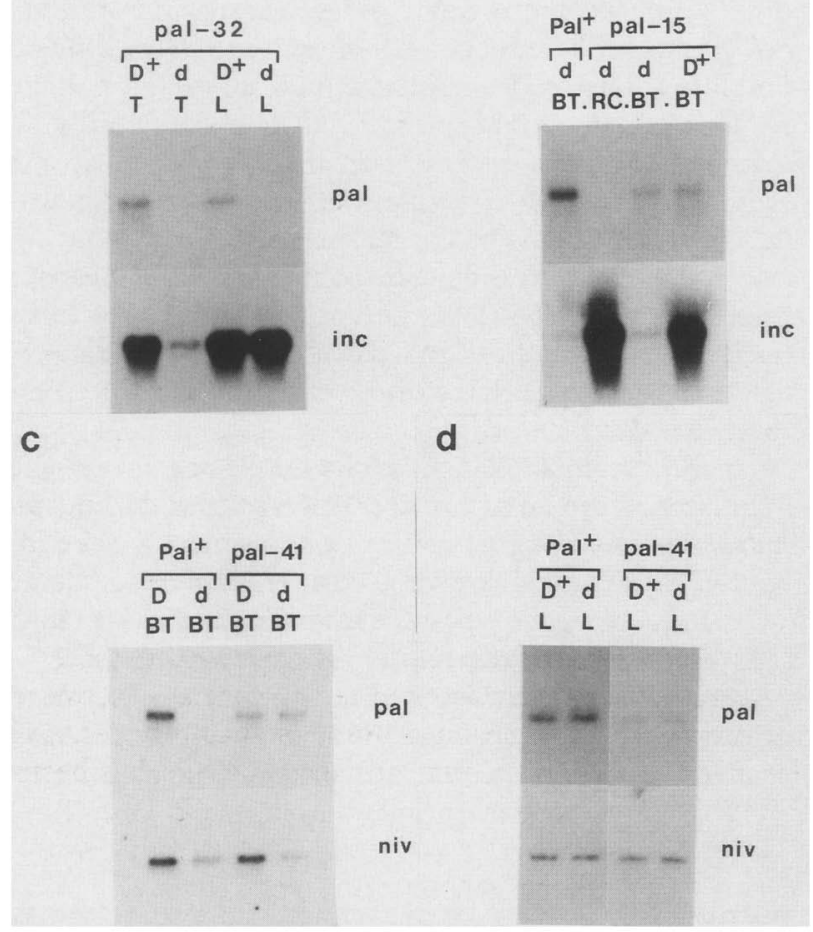

Figure 6. Effect of del on the expression of pal alleles. Northern blots probed with pal and inc or niv are shown. RNA was from flowers containing the pal alleles indicated, in a $\mathrm{Del}^{+}$ $\left(\mathrm{D}^{+}\right)$or del (d) background. The areas of the flowers from which RNA was extracted are indicated above each lane (for details, see Fig. 2). $(a$ and $b)$ Each lane contains 3 grams of poly $|\mathbf{A}|^{+}$ RNA; $(c$ and $d) 2 \mu \mathrm{g}$ of total RNA was used.

placed by a new sequence as a result of a large-scale chromosomal inversion (Robbins et al. 1989) (Fig. 5). This allele produces $\sim 25 \%$ of the level of transcript produced by $\mathrm{Pal}^{+}$. The levels of pal transcript at the base of the tubes and lobes in flowers containing pal-41 were similar in $\mathrm{Del}^{+}$or del backgrounds (Fig. 6c,d). As expected, the control showed that niv was regulated by del in pal-41 genotypes. These results show that pal expression in the tubes and lobes of pal-41 is independent of del and confirm that region $\mathrm{C}$ is required for the interaction between pal and del.

\section{Discussion}

We have shown that different patterns of expression conferred by the pal alleles reflect the action of three cis-acting upstream regions, A, B, and C. Region A is a 7-bp sequence containing the palindrome CACGTG, which is identical to the well-characterized upstream regulatory element of the adenovirus-2 major late promoter (Yu and Manley 1984; Carthew et al. 1985) and to the core sequence of the ' $G$ ' box, which has been found in several plant genes and shown to bind a nuclear factor (Giuliano et al. 1988; Shulze-Lefert et al. 1989). A protein specifically binding to this hexamer has also been described in A. majus (D. Steiger, H. Kaulen, and J. Schell, pers. comm.). Region B is 9 bp long and contains the sequence TTCGCG, which is identical to the core of the upstream element of the adenovirus-5 EIIA-E promoter (SivaRaman and Thimmappaya 1987). Region C is $79 \mathrm{bp}$ long and includes the CAAT box (Coen et al. 1986). The effects of these regions on expression, taken together with their structures and position, suggest that they most probably bind transcription factors. Similar multiple cis-acting upstream regions have been identified for many other genes by in vitro mutagenesis and transformation. However, unlike the analysis presented here, these studies assay gene expression in an abnormal chromosomal environment and subtle differences in pattern expression are not easily addressed (Fluhr et al. 1986; Dean et al. 1988; Willmitzer 1988).

The altered patterns of expression produced by deletion of the $A, B$, and $C$ regions suggest that they interact directly or indirectly with a prepattern of spatially distributed genetic signals. To determine the nature of these interactions, we analyzed how the deletions affected regulation of pal by a trans-acting gene, del. In del mutants, the level of pal expression in the tubes in reduced to $1 \%$ of wild type, but the level in lobes is unaffected. In affecting expression of anthocyanin genes in particular regions, del resembles the $c 1$ locus of maize, which is thought to encode a transcription activator controlling gene expression only in the aleurone and scutellum (Chen and Coe 1977; Cone et al. 1986; PazAres et al. 1987). We have shown that deletion of A, B, or $\mathrm{C}$ can alter the interaction between pal and del in two different ways. First, the deletion of $\mathrm{A}$ or $\mathrm{AB}$ brings pal under the regulation of del in the lobes. Second, deletion of $\mathrm{ABC}$ uncouples pal from regulation by del. How do these observations help to explain diverse patterns of $\mathrm{pal}$ expression resulting from the deletions?

The pal alleles that lack regions $\mathrm{A}$ or $\mathrm{AB}$ are regulated by $d e l$ in the lobes as well as the tubes, suggesting that the $\mathrm{Del}^{+}$gene product is present in both of these areas of the flower. When $\mathrm{AB}$ is present, as in $\mathrm{Pal}^{+}$alleles, del only affects pal expression in the tubes, suggesting that there is a mechanism that masks the effect of del in the lobes. We propose that this masking is the result of the presence of lobe-specific factors. In the absence of an active $\mathrm{Del}^{+}$product (D), there are presumably one or more factors (L) responsible for maintaining the wild-type levels of $\mathrm{Pal}^{+}$expression in the lobes. If both $\mathrm{D}$ and $\mathrm{L}$ are transcription activators that interact with the same site, and $\mathrm{L}$ has a much higher affinity than $\mathrm{D}$ for the site or is more efficient at activating transcription than $D$, removal of $D$ will have little or no effect on transcription (see Fig. 7). If deletion of $A B$ affected the interaction of pal with $\mathrm{L}$ much more than with $\mathrm{D}$, an effect of $\mathrm{D}$ on the 
$\mathrm{Pal}^{+}$
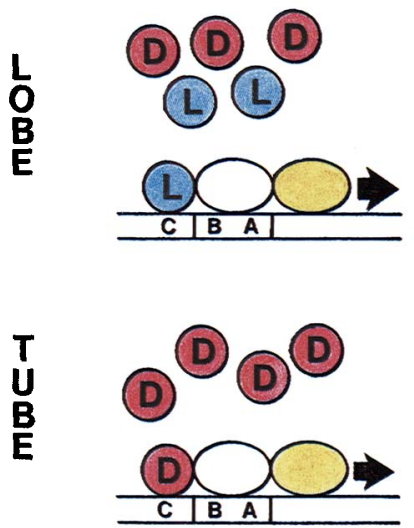

pal-32
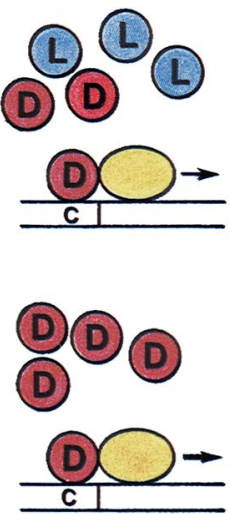

Figure 7. Model for the spatial patterns of expression of $\mathrm{Pal}^{+}$ and pal-32. The $\mathrm{D}$ factor is represented by red circles, and the $\mathrm{L}$ factor, by blue circles. Open ellipses represent factors interacting with regions $A B$, and yellow ellipses represent factors binding to the region proximal to the transcription initiation site (to the right of $\mathrm{AB}$ ). Arrows indicate the levels of pal expression (for details, see text).

lobes would become apparent because $\mathrm{D}$ would no longer be efficiently masked by L. Such a model also explains why the $A B$ deletion reduces transcription in the lobes more than in the tubes. According to this view, the $\mathrm{AB}$ region binds common rather than spatially specific transcription factors, and its effect on spatial pattern results from altered interactions between these common factors and spatially distributed factors such as L. A precedent for this type of model is that proposed for the regulation of the immunoglobulin genes (Staudt et al. 1986; Scheidereit et al. 1987). In this case, two transcription factors bind to the same octamer, but only one of the factors is bound in B lymphocytes, even though both factors are present. It has been suggested that other factors, which need not be tissue specific, interact differentially with the octamer-binding transcription factors and thus determine which interaction occurs.

Alleles lacking region $\mathrm{ABC}$ are uncoupled from regulation by del, suggesting that region $\mathrm{C}$ binds either $\mathrm{D}$ or a protein that interacts with $D$. Interestingly, region $C$ contains a CAAT box, which is a sequence that has been shown to interact with families of diverse factors (Dorn et al. 1987). Therefore, this may be a candidate for the site of action of D and L, although other possibilities cannot be excluded because region $C$ is 79 bp long. In the absence of regions $\mathrm{ABC}$, pal expression is restricted to the base of the tube at $\sim 1 \%$ of the wild-type level. Presumably, there are factors that activate pal expression at the base of the tube, which act in combination with factors bound in regions $A B C$ to give wild-type levels of expression in this area.

An inversion that replaces $\mathrm{ABC}$ and all sequences farther upstream by a new sequence gives a very different pattern of expression from the other alleles. This allele is also uncoupled from regulation by del, but its expression at the base of the tube is $>20$-fold greater than alleles lacking only region $\mathrm{ABC}$ and is also much higher than $\mathrm{Pal}^{+}$; del genotypes. Therefore, the pattern of expression cannot be explained simply by the loss of sequences involved in activation of transcription. Either the inversion removes transcription inhibitory sequences located $>190$ bp upstream or it introduces novel regulatory sequences (Coen et al. 1988; Robbins et al. 1989). In either case, this allele reveals a further spatial pattern of factors that can potentially interact with pal.

The results discussed above suggest that $\mathrm{Pal}^{+}$expression results from an interaction of the upstream region with a prepattern of multiple common and spatially specific transcription factors in the flower. Studies in other systems indicate that multiple interactions between transcription factors in different areas or tissues are a general feature of eukaryotic genes. This suggests that most genes could potentially have multiple alleles with different patterns of expression analogous to the pal alleles, providing a very large source of genetic variation. How have such complex regulatory systems evolved? Two mechanisms could result in a gene coming under the control of a novel transcription factor. The binding specificity of the factor could change so that it recognized an upstream sequence of the gene. However, this would have pleiotropic consequences because it may uncouple the genes already regulated by the factor and it may also bring other genes under the control of the factor. A more likely mechanism, without these pleiotropic effects, is that the upstream region of the gene could mutate to a sequence recognized by the factor, hence, bringing the gene under its control. This suggests that changes in the upstream regions of genes may be the most common and flexible way for regulatory systems to evolve and may account for the current interaction of multiple factors with these regions. This is supported by the observation that interspecific differences in the regulation of the alcohol dehydrogenase (ADH) gene of Drosophila are the result of cis-acting mutations in the $\mathrm{ADH}$ gene (Dickinson and Carlson 1979; Brennan et al. 1988). The different patterns of expression of the pal alleles may therefore reflect the complex evolutionary history of the pal upstream region.

\section{Materials and methods}

Antirrhinum stocks

pal alleles have the same numbers as the lines in which they are maintained. pal lines JI501, JI520, JI518, JI33, JI32, JI15, and JI41 and the standard wild-type JI7 have been described previously (Fincham and Harrison 1967; Coen et al. 1986). Allele pal-35G was obtained from Gatersleben, courtesy of Dr. C. Lehmann. The line containing pal-35G was also mutant for del. Allele pal-35G was transferred into a $\mathrm{Del}^{+}$background by crossing to JI 15. The pal-569 allele was from a 'Kimosy white' line, kindly supplied by J.M. Pfister (Pfister and Widholm 1984; Martin et al. 1987). Kimosy white lacks any anthocyanin pigment because it also carries a del mutation. In a $\mathrm{Del}^{+}$background, pal-569 confers pigmentation restricted to a ring at the 
base of the tube, similarly to pal-15. J18 carries a del allele in an otherwise wild-type background and shares a common origin with JI7. Lines containing pal-15, pal-32, or pal-41 in a del background were obtained from crosses between JI15, JI32, or JI41 and the del line JI8. Plants carrying pal-33 in a del background were obtained by crossing JI33 with another del line, JI6, also wild type with respect to other genes of the anthocyanin pathway.

\section{Molecular analysis}

The methods for anthocyanin, RNA, and DNA analysis have been described previously (Coen et al. 1986; Robbins et al. 1989|. The probes used in slot-blots and Northern blots were a 1.4-kb EcoRI-HindIII fragment of the pal clone pIAM4 (Coen et al. 1986), a $5.7-\mathrm{kb}$ EcoRI fragment of the niv clone pAm3 (Wienand et al. 1982), and a cDNA clone of the inc gene (C. Martin, unpubl.). The blots were first probed with pal and then with either niv or inc after washing the pal signal with $0.1 \times$ SSC, $0.5 \%$ SDS at $70^{\circ} \mathrm{C}$. The pal alleles were analyzed by Southern blotting using a 4.3-kb EcoRI fragment of the pal clone pJAM501 (Coen et al. 1986). For sequence analysis, each of the pal alleles was cloned into $\lambda$ NM1149 (Murray 1982), as described previously (Coen et al. 1986). A HindIII fragment containing the site of excision of Tam 3 was isolated from each of the clones, ligated into M13mp19 (Norrander et al. 1983), and sequenced by the dideoxy chain-termination method (Sanger et al. 1977).

\section{Acknowledgments}

We thank John Firmin for synthesizing leucocyanidin, Hans Sommer for providing pAm3, Andrew Davies and Peter Scott for photography, and David Hopwood and Caroline Dean for constructive comments on the manuscript.

\section{References}

Baur, E. 1924. Untersuchungen Uber das Wesen, die Enslehung und die vererbung von Rassenunterschieden bei Antirrhinum maius. Bibl. Genet. 4: 1-70.

Brennan, M.D., C.Y. Wu, and A.J. Berry. 1988. Tissue-specific differences for the alcohol dehydrogenase genes of Hawaiian Drosophila are conserved in Drosophila melanogaster transformants. Proc. Natl. Acad. Sci. 85: 6866-6869.

Campuzano, S., L. Carramolino, C.V. Cabrera, M. Ruiz-Gomez, R. Villares, A. Boronat, and J. Modolell. 1985. Molecular genetics of the achaete-scute gene complex of $D$. melanogaster. Cell 40: 327-338.

Carthew, R.W., L.A. Chodosh, and P.A. Sharp. 1985. An RNA polymerase II transcription factor binds to an upstream element in the adenovirus major late promoter. Cell 43: 439448.

Chen, S.M. and E.H. Coe, Jr. 1977. Control of anthocyanin synthesis by the $C$ locus in maize. Biochem. Genet. 15: 333346.

Chia, W., G. Howes, M. Martin, Y. Meng, K. Moses, and S. Tsubota. 1986. Molecular analysis of the yellow locus of Drosophila. EMBO I. 5: 3597-3605.

Coen, E.S. and R. Carpenter. 1986. Transposable elements in Antirrhinum majus: Generators of genetic diversity. Trends Genet. 20: 292-296.

1988. A semi-dominant allele, niv-525, acts in trans to inhibit the expression of its wild-type homologue in Antirrhinum majus. EMBO I. 7: 877-883.
Coen, E.S., R. Carpenter, and C. Martin. 1986. Transposable elements generate novel spatial patterns of gene expression in Antirrhinum majus. Cell 47: 285-296.

Coen, E.S., J. Almeida, T.P. Robbins, A. Hudson, and R. Carpenter. 1988. Molecular analysis of genes determining spatial patterns in Antirrhinum majus. In Temporal and spatial regulation of plant genes (ed. D.P.S. Verma and R.B. Goldberg), pp. 63-82. Springer-Verlag, Wien, New York.

Coen, E.S., T.P. Robbins, J. Almeida, A. Hudson, and R. Carpenter. 1989. Consequences and mechanisms of transposition in Antirrhinum majus. In Mobile DNA /ed. D. Berg and M. Howe), pp. 411-434. American Society for Microbiology. Washington, D.C.

Cone, K.C., F.A. Burr, and B. Burr. 1986. Molecular analysis of the maize anthocyanin regulatory locus $C 1$. Proc. Natl. Acad. Sei. 83: 9631-9635.

Davision, D., C.H. Chapman, C. Wedeen, and P.M. Bingham. 1985. Genetic and physic studies of a portion of the white locus participating in transcriptional regulation and in synapsis-dependent interactions in Drosophila adult tissues. Genetics 110: 479-494.

Dean, C., J. Jones, M. Favreau, P. Dunsmuir, and J. Bedbrook. 1988. Influence of flanking sequences on variability in expression levels of an introduced gene in transgenic tobacco plants. Nucleic Acids Res. 16: 9267-9283.

Dickinson, W.J. and H.L. Carlson. 1979. Regulation of the tissue specificity of an enzyme by a cis-acting genetic element: Evidence from interspecific Drosophila hybrids. Proc. Natl. Acad. Sci. 76: 4559-4562.

Dorn, A., J. Bollekens, A. Staub, C. Benoist, and D. Mathis. 1987. A multiplicity of CCAAT box-binding proteins. Cell 50: $863-872$.

Fincham, J.R.S. and B. Harrison. 1967. Instability at the Pal locus in Antirrhinum majus. II. Multiple alleles produced by mutation of one original unstable allele. Heredity 22: $211-227$.

Fluhr, R., C. Kuhlemeier, and N.-H. Chua. 1986. Organ-specific and light-induced expression of plant genes. Science 232: $1106-1112$.

Forkmann, G. and G. Stotz. 1981. Genetic control of flavanone3-hydroxylase and flavonoid-3'-hydroxylase activity in $A n$ tirrhinum majus (snapdragon). Z. Naturforsch. 36c: 411416.

Geyer, P.K., M.M. Green, and V.C. Corces. 1988. Reversion of a gypsy-induced mutation at the yellow locus of Drosophila melanogaster is associated with the insertion of a newly defined transposable element. Proc. Natl. Acad. Sci. 85: 39283942.

Giuliano, G., E. Pichersky, V.S. Malik, M.P. Timko, P.A. Scolnik, and A.R. Cashmore. 1988. An evolutionary conserved protein binding sequence upstream of a plant lightregulated gene. Proc. Natl. Acad. Sci. 85: 7089-7093.

Heller, W., G. Forkmann, L. Britsch, and H. Grisebach. 1985. Enzymatic reduction of $(+1$-dihydroflavonols to flavan-3,4cis-diols with flower extracts from Matthiola incana and its role in anthocyanin biosynthesis. Planta 165: 284-287.

Kristiansen, K.N. 1984. Biosynthesis of proanthocyanidins in barley: Genetic control of the conversion of dihydroquercetin to catechin and procyanidins. Carlsberg Res. Commun. 49: 503-524.

Martin, C.R., R. Carpenter, E.S. Coen, and A.G.M. Gerats. 1986. The control of floral pigmentation in Antirrhinum majus. Soc. Exp. Biol. Symp. 32: 19-52.

Martin, C.R., R. Carpenter, H. Sommer, H. Saedler, and E.S. Coen. 1985. Molecular analysis of instability in flower pigmentation in Antirrhinum majus, following isolation of the 
pallida locus by transposon tagging. EMBO /. 4: 1625-1630.

Martin, C., E. Coen, T.P. Robbins, J. Bartlett, J. Almeida, and R. Carpenter. 1987. The control of floral pigmentation in $A n-$ tirrhinum. Biochem. Soc. Trans. 15: 14-17.

Murray, N.E. 1982. Phage lambda and molecular cloning. In Lambda II (ed. R.W. Hendrix, J.W. Roberts, F.W. Stahl, and R.A. Weissberg/, pp. 395-442. Cold Spring Harbor Laboratory, Cold Spring Harbor, New York.

Norrander, J., T. Kempe, and J. Messing. 1983. Construction of improved vectors using oligodeoxynucleotide-directed mutagenesis. Gene 26: 101-106.

Paz-Ares, J., D. Ghosal, U. Wienand, P.A. Peterson, and H. Saedler. 1987. The regulatory $\mathrm{cl}$ locus of Zea mays encodes a protein with homology to myb proto-oncogene products and with structural similarities to transcriptional activators. $E M B O$ I. 6: 3553-3558.

Pfister, J.M. and J.M. Widholm. 1984. Plant regeneration from snapdragon tissue cultures. Hortscience 19(6): 852-854.

Pirrotta, V., H. Steller, and M.P. Bozzetti. 1985. Multiple upstream regulatory elements control the expression of the Drosophila white gene. EMBO f. 4: 3501-3508.

Robbins, T.P., R. Carpenter, and E.S. Coen. 1989. A chromosome rearrangement suggests that donor and recipient sites are associated during Tam 3 transposition in Antirrhinum maius. EMBO \%. 8: 5-13.

Sanger, F., S. Nicklen, and A.R. Coulson. 1977. DNA sequencing with chain-terminating inhibitors. Proc. Natl. Acad. Sci. 74: 5463-5467.

Scheidereit, C., A. Heguy, and R.G. Roeder. 1987. Identification and purification of a human lymphoid-specific octamerbinding protein (OTF-2) that activates transcription of an immunoglobulin promoter in vitro. Cell 51: 783-793.

Schulze-Lefert, P., J. Dangl, M. Becker-Andre, K. Hahlbrock, and W. Schulz. 1989. Inducible in vivo DNA footprints define sequences necessary for UV light activation of the parsley chalcone synthase gene. EMBO I. 8: 651-656.

Schwarz-Sommer, Z., N. Shepherd, E. Tacke, A. Gierl, W. Rohde, L. Leclercq, M. Mattes, R. Berndtgen, P.A. Peterson, and $\mathrm{H}$. Saedler. 1987. Influence of transposable elements on the structure and function of the $A 1$ gene of Zea mays. $E M B O$ I. 6: 287-294.

SivaRaman, L. and B. Thimmappaya. 1987. Two promoter-specific host factors interact with adjacent sequences in an EIA-inducible adenovirus promoter. Proc. Natl. Acad. Sci. 84: 6112-6116.

Staudt, L.M., H. Singh, R. Sen, T. Wirth, P.A. Sharp, and D. Baltimore. 1986. A lymphoid-specific protein binding to the octamer motif of immunoglobulin genes. Nature 323: 640643.

Stern, C. 1954. Two or three bristles. Am. Sci. 42: 213-247.

1968. Genetic mosaics and other essays, Harvard University Press, Cambridge.

Stickland, R.G. and B.J. Harrison. 1974. Precursors and genetic control of pigmentation. I. Induced biosynthesis of pelargonidin, cyanidin, and delphinidin in Antirrhinum majus. Heredity 33: $108-112$.

Wheldale, M. 1907. The inheritance of flower colour in Antirrhinum majus. Proc. Roy. Soc. London Ser. B. 79: 288-305.

Wienand, U., H. Sommer, Z. Schwarz, N. Shepherd, H. Saedler, F. Kreutzaler, H. Ragg, E. Fautz, K. Hahlbrock, B.J. Harrison, and P.A. Peterson. 1982. A general method to identify plant structural genes among genomic DNA clones using transposable element induced mutations. Mol. Gen. Genet. 187: 195-201.

Willmitzer, L. 1988. The use of transgenic plants to study plant gene expression. Trends Genet. 4: 13-18.
Yu, Y.T. and J.L. Manley. 1984. Generation and functional analyses for base-substitution mutants of the adenovirus 2 major late promoter. Nucleic Acids Res. 12: 9309-9321. 


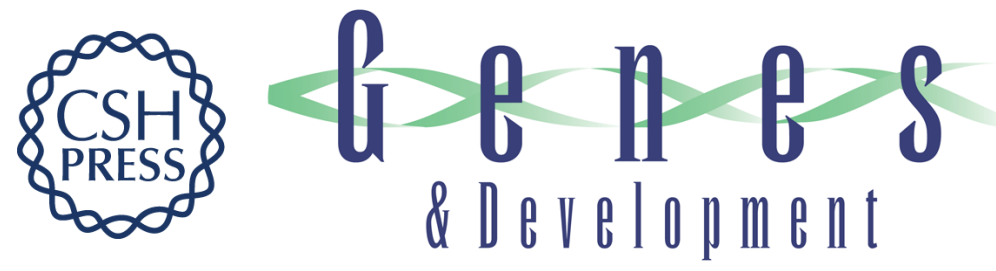

\section{Genetic interactions underlying flower color patterns in Antirrhinum majus.}

J Almeida, R Carpenter, T P Robbins, et al.

Genes Dev. 1989, 3:

Access the most recent version at doi:10.1101/gad.3.11.1758

References This article cites 41 articles, 10 of which can be accessed free at:

http://genesdev.cshlp.org/content/3/11/1758.full.html\#ref-list-1

License

Email Alerting

Service

Receive free email alerts when new articles cite this article - sign up in the box at the top right corner of the article or click here.

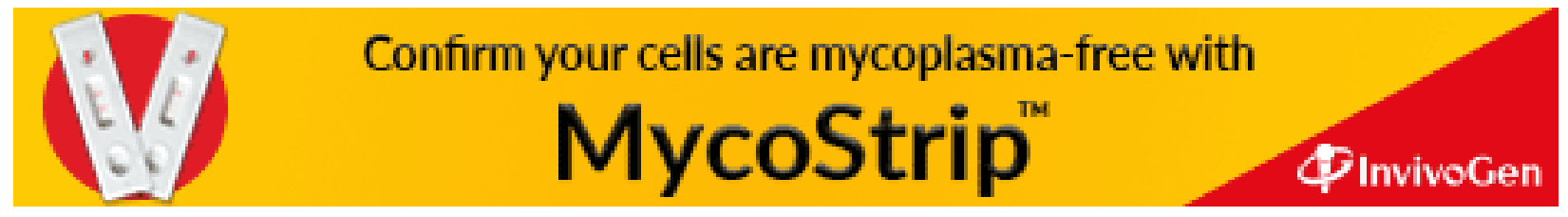

